\title{
HOMOLOGY WITH MULTIPLE-VALUED FUNCTIONS APPLIED TO FIXED POINTS
}

\author{
BY \\ RICHARD JERRARD
}

\begin{abstract}
Certain multiple-valued functions ( $m$-functions) are defined and a homology theory based upon them is developed. In this theory a singular simplex is an $\boldsymbol{m}$-function from a standard simplex to a space and an $\boldsymbol{m}$-function from one space to another induces a homomorphism of homology modules.

In a family of functions $f_{x}: Y \rightarrow Y$ indexed by $x \in X$ the fixed points of $f_{X}$ are taken to be the images at $x$ of a multiple-valued function $\phi: X \rightarrow Y$. In certain circumstances $\phi$ is an $m$-function, giving information about the behavior of the fixed points of $f_{X}$ as $x$ varies over $X$. These facts are applied to selfmaps of products of compact polyhedra and the question of whether such a product has the fixed point property for continuous functions is essentially reduced to the question of whether one of its factors has the fixed point property for $m$-functions. Some light is thrown on the latter problem by using the homology theory to prove a Lefschetz fixed point theorem for $m$-functions.
\end{abstract}

1. Introduction. In this paper we define certain multiple-valued functions called $m$-functions, and apply them with a homology theory based upon them to prove theorems about the fixed point property (f.p.p.) for product spaces. We also obtain information on the behaviour of fixed points during a homotopy.

The problem of which product spaces have the f.p.p. is an old one, first posed by Kuratowski [7] in 1930. Various results have been obtained by Fadell [3], Lopez [8], Knill [6] and others, mostly in the form of counterexamples. We obtain a positive theorem $(3.6,3.7)$ and as one consequence some results for polyhedra (6.2 and corollaries).

In the outline the procedure is as follows. In $\S 2$ we define $m$-functions, show that with Hausdorff spaces they form a category, and give some properties summarized in 2.18. In $\S 3$ we show that if $f: X \times Y \rightarrow X \times Y$, then under suitable conditions the set of points in $X \times Y$ whose $Y$-coordinate is unchanged by $f$ forms the graph of an $m$-function $\phi: X \rightarrow Y$; similarly the points whose $X$-coordinate is unchanged yield an $m$-function $\psi: Y \rightarrow X$. If these two graphs intersect, then $f$ has a fixed point. By taking the composition $\xi_{f}=\psi \circ \phi: X$ $\rightarrow X$ we reduce the question of whether $f$ has a fixed point in $X \times Y$ to the

Received by the editors April 12, 1974 and, in revised form, January 16, 1975. AMS (MOS) subject classifications (1970). Primary 55C20; Secondary 55B10, $54 \mathrm{C} 60$. 
question of whether $\xi_{f}$ has a fixed point in $X$. This is essentially the content of 3.6 and 3.7 .

Since $\xi_{f}$ is an $m$-function, the question arises as to when a space $X$ has the f.p.p. for $m$-functions (the $m$-f.p.p.). To this end in $\$ 4$ we define an $m$-homology theory, a homology theory using $m$-functions. This is a generalization of ordinary singular homology, and the point is to prove a Lefschetz fixed point theorem for $m$-functions, which is done in $\$ 5$. We do not give any other applications of $m$ homology, but it is interesting because the set of functions on spaces has been widened to include $m$-functions, and the set of induced homology module homomorphisms has been correspondingly enlarged.

In $\$ 6$ we have available a Lefschetz theorem which throws some light on the $m$-f.p.p. This is used in conjunction with 3.6 to prove theorems on the fixed point property for products of polyhedra.

I am indebted to the University of Cambridge for the use of facilities during 1972-73, and to the referee for suggesting several clarifications.

2. $m$-functions. In this section we shall define and give some of the properties of certain multiple-valued functions to be called $m$-functions. They were defined in a different way and used in [4] and [5]. The present definition was suggested by William L. Young. It is given below, and the properties of $m$-functions are summarized in 2.18 .

If $X$ and $Y$ are Hausdorff spaces and $A$ is a ring without zero divisors, an $m$-function from $X$ to $Y$ over $A$ is a multiple-valued function from a subset of $X$ to $Y \times A$ satisfying conditions given below. We shall use the notation $f: X \rightarrow$ $Y \times A$, bearing in mind that the domain of $f$ may not be all of $X$ (it may even be empty). If $p_{1}: Y \times A \rightarrow Y$ is the projection, we denote the composition $p_{1} \circ f$ by $f^{\prime}: X \rightarrow Y$, a multiple-valued function whose domain may be a proper subset of $X$. Here $f^{\prime}$ may be regarded as a set of ordered pairs, with

$$
f^{\prime}(x)=\left\{y \mid(x, y) \in f^{\prime}\right\}
$$

where $f^{\prime}$ is used to denote both this function and its graph.

Each $m$-function $f: X \rightarrow Y \times A$ is defined by its defining function $\tilde{f}: X \times$ $Y \rightarrow A$ which is single-valued and has domain $X \times Y$. If $\tilde{f}$ is given, then as a set of ordered pairs

$$
f^{\prime}=\operatorname{cl}\{(x, y) \in X \times Y \mid \widetilde{f}(x, y) \neq 0\},
$$

where cl denotes closure. We then write

$$
f(x)=\left\{(y, \widetilde{f}(x, y)) \in Y \times A \mid y \in f^{\prime}(x)\right\} .
$$

In working with $m$-functions we shall not use this expression, but will return to the defining function $\widetilde{f}$. We regard $f$ as a multiple-valued function from a subset 
of $X$ to $Y$ (namely $f^{\prime}$ ) in which each ordered pair $(x, y)$ has been tagged with an element of $A$ (namely $\widetilde{f}(x, y)$ ). The multiple-valued function $f^{\prime}$ is simply the untagged version of $f$.

Definition 2.1. $f: X \rightarrow Y \times A$ is an $m$-function defined by the defining function $\widetilde{f}: X \times Y \rightarrow A$ if $\widetilde{f}$ has the following properties:

(1) for all $x \in X, f^{\prime}(x)$ is a finite or empty subset of $Y$;

(2) if $f^{\prime}\left(x^{\prime}\right)=\left\{y_{1}, y_{2}, \ldots, y_{n}\right\}$ there exist disjoint neighborhoods $\hat{V}_{i}\left(y_{i}\right)$ such that if $V_{i}\left(y_{i}\right) \subset \hat{V}_{i}$ there exists a neighborhood $U\left(x^{\prime}\right)$ satisfying

(a) $\Sigma_{y \in V_{i}} \widetilde{f}(x, y)=\widetilde{f}\left(x^{\prime}, y_{i}\right)$ for all $x \in U, i=1,2, \ldots, n$;

(b) $\widetilde{f}(x, y)=0$ for $x \in U$ and $y \in\left[Y-\bigcup_{i=1}^{n} V_{i}\right]$; if $f^{\prime}\left(x^{\prime}\right)=\varnothing$ there exists a neighborhood $U\left(x^{\prime}\right)$ such that $\tilde{f}(x, y)=0$ for all $x \in U$.

Definition 2.2. The multiplicity of a point $(x, y) \in f^{\prime}$ is $\tilde{f}(x, y)$. The multiplicity of the m-function $f$ at $x$ is $\Sigma_{y \in Y} \widetilde{f}(x, y)$. In the following lemma we show that if $X$ is connected, the multiplicity of $f$ is independent of the point $x$ at which it is evaluated.

$$
\begin{aligned}
& \text { LEMMA 2.3. If } X \text { is connected and } x_{1}, x_{2} \in X \text {, then } \Sigma_{y \in Y} \widetilde{f}\left(x_{1}, y\right)= \\
& \Sigma_{y \in Y} \widetilde{f}\left(x_{2}, y\right) \text {. }
\end{aligned}
$$

Proof. We define $m: X \rightarrow A$ by $m(x)=\Sigma_{y \in Y} \widetilde{f}(x, y)$. If the statement is false there exists a point $x^{\prime} \in X$ such that $m\left(x^{\prime}\right)=a$ and for each neighborhood $U\left(x^{\prime}\right)$ there exists $x \in U$ such that $m(x) \neq a$. If $f^{\prime}\left(x^{\prime}\right)=\left\{y_{1}, y_{2}, \ldots, y_{m}\right\}$ there exist neighborhoods $V_{i}\left(y_{i}\right), \hat{U}\left(x^{\prime}\right)$ such that if $x \in \hat{U}$,

$$
\sum_{y \in Y} \widetilde{f}(x, y)=\sum_{i=1}^{m} \sum_{y \in V_{i}} \widetilde{f}(x, y)=\sum_{i=1}^{m} \widetilde{f}\left(x^{\prime}, y_{i}\right)=a
$$

if $f^{\prime}\left(x^{\prime}\right)=\varnothing$ then $a=0$ and $\Sigma_{y \in Y} \widetilde{f}(x, y)=0=a$ for all $x$ in some neighborhood of $x^{\prime}$. In both cases we have a contradiction.

We can now speak of the multiplicity of the m-function $f$,

$$
m(f)=\sum_{y \in Y} \widetilde{f}(x, y),
$$

which is well defined if $x$ is connected.

We next note that if $Y$ is compact, then condition (2b) of Definition 2.1 is automatically satisfied.

Proposition 2.4. If $Y$ is compact and $\tilde{f}$ satisfies conditions (1) and (2a) of the m-function definition, then it also satisfies condition (2b).

Proof. Choosing $x^{\prime} \in X$ we have from condition (1) that $f^{\prime}\left(x^{\prime}\right)=\left\{y_{1}, y_{2}\right.$, $\ldots, y_{n}$ \}. From condition (2a) we obtain neighborhoods $\hat{V}_{i}\left(y_{i}\right), U(x)$. Since $f^{\prime} \subset X \times Y$ is closed, for $y \in\left[Y-\cup_{i} \hat{V}_{i}\right]$ there is a neighborhood $W\left(x^{\prime}, y\right)$ such that $W \cap f^{\prime}=\varnothing$. Then 


$$
\left[\bigcup_{i}\left(U \times \hat{V}_{i}\right)\right] \cup\left[\bigcup_{y \in\left[Y-U \hat{V}_{i}\right]} W\left(x^{\prime}, y\right)\right]=V \supset\left[\left\{x^{\prime}\right\} \times Y\right]
$$

and by compactness of $Y$ there exists a neighborhood $\hat{U}\left(x^{\prime}\right)$ such that $\hat{U} \times Y \subset V$. The neighborhood $\hat{U}$ satisfies condition (2b).

We consider next the composition of $m$-functions. If $m$-functions $f: X \rightarrow$ $Y \times A$ and $g: Y \rightarrow Z \times A$ are given by defining functions $\tilde{f}$ and $\tilde{g}$, we can use them to produce the defining function $\tilde{g} \widetilde{\circ} \tilde{f}$ of the $m$-function $g \circ f$.

DEFinition 2.5. If $f: X \rightarrow Y \times A$ and $g: Y \rightarrow Z \times A$ are $m$-functions over $A$, their composition $g \circ f: X \rightarrow Z \times A$ is defined by the function

$$
\tilde{g} \circ \tilde{f}: X \times Z \rightarrow A, \quad(x, z) \mapsto \sum_{y \in Y} \widetilde{f}(x, y) \tilde{g}(y, z) .
$$

Proposition 2.6. The composition of m-functions is an m-function, and $m(g \circ f)=m(f) m(g)$.

Proof. To prove property (1) of the definition, choose $x \in X$ and write $f^{\prime}(x)=\left\{y_{1}, y_{2}, \ldots, y_{n}\right\}$. Then $g^{\prime}\left(y_{i}\right)=\left\{z_{i 1}, z_{i 2}, \ldots, z_{i m(i)}\right\}$ and we claim that $g^{\prime} \circ f^{\prime}(x) \subset \bigcup_{i} g^{\prime}\left(y_{i}\right)$, a finite set. To prove this, suppose that $z \in g^{\prime} \circ f^{\prime}(x)$ and $z \notin \bigcup_{i} g^{\prime}\left(y_{i}\right)$. By property (2) for $\widetilde{f}$, there exist neighborhoods $\hat{V}_{i}\left(y_{i}\right)$ satisfying certain conditions. Also, since $g^{\prime}$ is a closed subset of $Y \times Z$, for each $i=$ $1,2, \ldots, n$ there exists a neighborhood $V_{i}\left(y_{i}\right) \times W(z)$ of $\left(y_{i}, z\right)$ such that $\left(V_{i} \times\right.$ W) $\cap g^{\prime}=\varnothing$ and $V_{i} \subset \hat{V}_{i}$. Then property (2) for $\tilde{f}$ gives a neighborhood $U(x)$ such that if $x^{\prime} \in U$ and $\widetilde{f}\left(x^{\prime}, y\right) \neq 0$ then $\chi \in U_{i} V_{i}$. But if $y \in U_{i} V_{i}$ and $z^{\prime} \in W(z)$ then $\tilde{g}\left(y, z^{\prime}\right)=0$. Therefore if $\left(x^{\prime}, z^{\prime}\right) \in U \times W$ we have $\widetilde{g} \sim \widetilde{f}\left(x^{\prime} z^{\prime}\right)$ $=0$. Hence $(x, z)$ is not in the closure of $\left\{\left(x^{\prime}, z^{\prime}\right) \mid \tilde{g} \sim \widetilde{f}\left(x^{\prime}, z^{\prime}\right) \neq 0\right\}$ and therefore $z \notin g^{\prime} \circ f^{\prime}(x)$.

To prove property (2a) for $\widetilde{g} \widetilde{\circ} \widetilde{f}$ we choose $\left(x_{0}, z_{0}\right) \in g^{\prime} \circ f^{\prime}$. There exists $\left\{y_{1}, y_{2}, \ldots, y_{m}\right\}$ such that $\left(x_{0}, y_{i}\right) \in f^{\prime},\left(y_{i}, z_{0}\right) \in g^{\prime}$. By property (2) for $\widetilde{f}$ there exist neighborhoods $\hat{V}_{i}\left(y_{i}\right)$, and for $\widetilde{g}(i=1,2, \ldots, m)$ there exist neighborhoods $\hat{W}_{i}\left(z_{0}\right)$; we put $\hat{W}\left(z_{0}\right)=\bigcap_{i} \hat{W}_{i}\left(z_{0}\right)$ and find neighborhoods $V_{i}\left(y_{i}\right)$ such that $V_{i} \subset \hat{V}_{i}$ and

$$
\sum_{z \in \hat{W}} \tilde{g}(y, z)=\tilde{g}\left(y_{i}, z_{0}\right) \quad \text { for all } y \in V_{i} \text {. }
$$

Returning to property (2) for $\widetilde{f}$, since $V_{i} \subset \hat{V}_{i}$ there exists a neighborhood $U\left(x_{0}\right)$ such that $\Sigma_{y \in V_{i}} \widetilde{f}(x, y)=\widetilde{f}\left(x_{0}, y_{i}\right)$ for all $x \in U$. We now consider $U \times \hat{W}$ and must show that

$$
\sum_{z \in \hat{W}} \tilde{g} \widetilde{\circ} \widetilde{f}(x, z)=\tilde{g} \approx \widetilde{f}\left(x_{0}, z_{0}\right) \text { for each } x \in U .
$$

We compute 


$$
\begin{aligned}
\sum_{z \in \hat{W}} \sum_{y \in Y} \tilde{f}(x, y) \tilde{g}(y, z)=\sum_{y \in Y}\left[\tilde{f}(x, y) \sum_{z \in \hat{W}} \tilde{g}(y, z)\right] \\
\quad=\sum_{i=1}^{m} \sum_{y \in V_{i}}\left[\tilde{f}(x, y) \sum_{z \in \hat{W}} \tilde{g}(y, z)\right] \quad \text { (using property (2b)) } \\
=\sum_{i=1}^{m} \sum_{y \in V_{i}}\left[\tilde{f}(x, y) \tilde{g}\left(y_{i}, z_{0}\right)\right]=\sum_{i=1}^{m} \tilde{f}\left(x_{0}, y_{i}\right) \tilde{g}\left(y_{i}, z_{0}\right) \\
=\tilde{g} \approx \tilde{f}\left(x_{0}, z_{0}\right) \quad \text { for each } x \in U .
\end{aligned}
$$

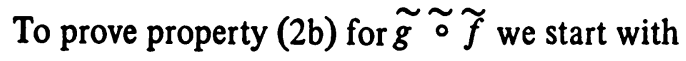

$$
f^{\prime}\left(x_{0}\right)=\left\{y_{1}, y_{2}, \ldots, y_{n}\right\}, \quad g^{\prime}\left(y_{i}\right)=\left\{z_{i 1}, z_{i 2}, \ldots, z_{i m(i)}\right\} .
$$

For each $y_{i}$ there exists by property (2b) for $\tilde{f}$ a neighborhood $\hat{V}_{i}\left(y_{i}\right)$. For each $z_{i j}, y_{i}$, there exists respectively by property (2) for $\tilde{g}$ a neighborhood $\hat{W}_{i j}\left(z_{i j}\right)$, $V_{i}\left(y_{i}\right)$ such that $V_{i} \subset \hat{V}_{i}$ and

$$
\tilde{g}(y, z)=0 \text { for } y \in V_{i}, z \notin \bigcup_{j} \hat{W}_{i j} \quad(i=1,2, \ldots, n) .
$$

Since $V_{i} \subset \hat{V}_{i}$ there exists a neighborhood $U\left(x_{0}\right)$ such that $\tilde{f}(x, y)=0$ for $x \in U$, $y \notin \bigcup_{i} V_{i}$. Now suppose that $x \in U, z \notin \bigcup_{i, j} \hat{w}_{i j}$. Then

$$
\tilde{\boldsymbol{g}} \sim \widetilde{f}(x, z)=\sum_{y \in Y} \tilde{f}(x, y) \tilde{g}(y, z)=\sum_{i=1}^{n} \sum_{y \in V_{i}} \tilde{f}(x, y) \tilde{g}(y, z)=0,
$$

for if $y \in V_{i}$ and $z \notin \bigcup_{j} \hat{w}_{i j}, \tilde{g}(y, z)=0$.

We must finally show that $m(g \circ f)=m(f) m(g)$. We have

$$
\begin{aligned}
m(g \circ f) & =\sum_{z \in Z} \sum_{y \in Y} \tilde{f}(x, y) \tilde{g}(y, z)=\sum_{y \in Y} \tilde{f}(x, y) \sum_{z \in Z} \tilde{g}(y, z) \\
& =\sum_{y \in Y} \tilde{f}(z, y) m(g)=m(f) m(g) .
\end{aligned}
$$

The restriction of an $m$-function to a subset is given by composition. If $B \subset X$ and $f: X \rightarrow Y \times A$, then $f \mid B: B \rightarrow Y \times A$ is $f \circ i$, where $i: B \rightarrow X$ is the injection.

We shall regard a continuous function $f: X \rightarrow Y$ as an $m$-function of multiplicity one. Its defining function is given by $\widetilde{f}(x, y)=1$ if $y=f(x)$ and $\widetilde{f}(x, y)$ $=0$ otherwise. With this convention the identity function $1_{X}: X \rightarrow X$ serves as an identity for $m$-functions, and if $f: X \rightarrow Y \times A$ is an $m$-function, $1_{Y} \circ f=f \circ$ $1_{X}=f$. It is also immediate from the definition that composition of $m$-function is associative. We have therefore proved 
THEOREM 2.7. Hausdorff spaces and $m$-functions over a ring $A$ form a category $M_{A}$.

Actually, the statement is true for all topological spaces. However, we will use only Hausdorff spaces in this paper, since this restriction will be needed later.

We now give some further properties of $m$-functions.

Turning to products, if $f: X \rightarrow Y \times A$ and $g: X \rightarrow Z \times A$ are $m$-functions over $A$, we can define the product m-function $h=f \times g: X \rightarrow Y \times Z \times A$ by the defining function

$$
\tilde{h}: X \times Y \times Z \rightarrow A, \quad(x, y, z) \mapsto \tilde{f}(x, y) \tilde{g}(x, z) .
$$

Proposition 2.8. Given m-functions $f: X \rightarrow Y \times A, g: X \rightarrow Z \times A$, then $f \times g: X \rightarrow Y \times Z \times A$ is an $m$-function and $m(f \times g)=m(f) m(g)$.

Proof. To prove property (1) of the definition we start with

$$
h^{\prime}=\operatorname{cl}\{(x, y, z) \mid \tilde{f}(x, y) \neq 0 \text { and } \tilde{g}(x, z) \neq 0\} .
$$

It follows that

$$
\begin{aligned}
& h^{\prime} \subset \operatorname{cl}\{(x, y, z) \mid \widetilde{f}(x, y) \neq 0\} \cap \operatorname{cl}\{(x, y, z) \mid \tilde{g}(x, z) \neq 0\}, \\
& h^{\prime} \subset\left(f^{\prime} \times Z\right) \cap\left(g^{\prime} \times Y\right),
\end{aligned}
$$

and since $f^{\prime}(x)$ and $g^{\prime}(x)$ are finite subsets of $Y$ and $Z, h^{\prime}(x)$ must be finite.

To prove property (2) we choose $\left(x_{0},\left(y_{0}, z_{0}\right)\right)$ such that $\left(y_{0}, z_{0}\right) \in h^{\prime}\left(x_{0}\right)$. By property (2) for $f$ and $g$ there exist neighborhoods $\hat{V}\left(y_{0}\right), \hat{W}\left(z_{0}\right), U(x)$ such that

$$
\sum_{y \in \hat{V}} \tilde{f}(x, y)=\widetilde{f}\left(x_{0}, y_{0}\right), \sum_{z \in \hat{W}} \tilde{g}(x, z)=\tilde{g}\left(x_{0}, z_{0}\right) \quad \text { for all } x \in U .
$$

Then if $x \in U$,

$$
\begin{array}{r}
\sum_{(y, z) \in \hat{V} \times \hat{W}} \tilde{f}(x, y) \tilde{g}(x, z)=\sum_{y \in \hat{V}} \tilde{f}(x, y) \sum_{z \in \hat{W}} \tilde{g}(x, z) \\
=\tilde{f}\left(x_{0}, y_{0}\right) \tilde{g}\left(y_{0}, z_{0}\right)=\tilde{h}\left(x_{0}, y_{0}, z_{0}\right) .
\end{array}
$$

The proof of property (2b) follows easily from the definitions as does $m(f \times g)$ $=m(f) m(g)$.

If $f: X \rightarrow Y \times A$ is an $m$-function, the ordered pairs $(x, y) \in f^{\prime}$ will be called points of $f$. They can be divided into two types.

Definition 2.9. A point $(x, y)$ of an $m$-function $f: X \rightarrow Y \times A$ is an ordinary point if $f^{\prime}$ is locally single-valued, that is, if there exist neighborhoods $U(x), V(y)$ such that $f^{\prime} \mid U: U \rightarrow V$ (both the domain and range of $f^{\prime}$ are 
restricted) is a function. Any point $(x, y)$ of $f$ that is not an ordinary point is a tangent point. If $f^{\prime} \subset X \times Y$ has the subspace topology, it is clear that the ordinary points form an open subset of $f^{\prime}$.

Proposition 2.10. If $\left(x^{\prime}, y^{\prime}\right)$ of $f: X \rightarrow Y \times A$ is an ordinary point, then $f^{\prime}$ is locally a continuous function whose multiplicity as an $m$-function is nonzero.

Proof. We denote $f^{\prime} \mid U: U \rightarrow V$ by $f_{r}^{\prime}$, where by hypothesis $f_{r}^{\prime}$ is a function, and suppose that $f_{r}^{\prime}\left(x^{\prime}\right)=y^{\prime}$. By property (2) there exists a neighborhood $\hat{V}\left(y^{\prime}\right)$ and we choose any $V^{\prime}\left(y^{\prime}\right) \subset \hat{V} \cap V$. Then there exists $U^{\prime}\left(x^{\prime}\right) \subset U$ such that

$$
\sum_{y \in V^{\prime}} \tilde{f}(x, y)=\widetilde{f}\left(x^{\prime}, y^{\prime}\right)=a \quad \text { for all } x \in U^{\prime} .
$$

We must have $a \neq 0$, for if $a=0$ then $\widetilde{f}(x, y)=0$ for all $(x, y) \in f^{\prime} \cap\left(U^{\prime} \times V^{\prime}\right)$ and $\left(x^{\prime}, y^{\prime}\right)$ cannot be in $\operatorname{cl}\{(x, y) \mid \tilde{f}(x, y) \neq 0\}$. It follows that if $x \in U^{\prime}$ then $f_{r}(x) \in V^{\prime}$, for if not the above sum would be zero.

We next consider the points of $f$ as a subset of $X \times Y$ (it is the set $f^{\prime} \subset X \times Y$ ) and separate it into its connected components. We show that each of these components defines a natural $m$-function. If $f_{1}^{\prime} \subset f^{\prime}$ is a component, then the component $m$-function $f_{1}: X \rightarrow Y \times A$ is defined by $\widetilde{f}_{1}: X \times Y \rightarrow A$ where

$$
\tilde{f}_{1}(x, y)= \begin{cases}\tilde{f}(x, y), & (x, y) \in f_{1}, \\ 0, & (x, y) \notin f_{1} .\end{cases}
$$

We must show that $f_{1}$ is in fact an $m$-function.

LEMMA 2.11. Given an m-function $f: X \rightarrow Y$, suppose that $\left(B_{1}, B_{2}\right)$ is a separation of $f^{\prime}$; that is, $B_{1} \cup B_{2}=f^{\prime}, B_{1} \cap \bar{B}_{2}=\bar{B}_{1} \cap B_{2}=\varnothing$. We define, for $j=1,2$,

$$
f_{j}: X \rightarrow Y \times A, \quad \tilde{f}_{j}(x, y)= \begin{cases}\tilde{f}(x, y), & (x, y) \in B_{j}, \\ 0, & (x, y) \notin B_{j} .\end{cases}
$$

Then $f_{1}$ and $f_{2}$ are $m$-functions.

Proof FOR $f_{1}$. Clearly $f_{1}$ satisfies properties (1) and (2b). For property (2a), first note that since $B_{1}$ is open in $f^{\prime}, B_{1}=B \cap f^{\prime}$ where $B$ is open in $X \times Y$. Now if $f_{1}^{\prime}\left(x_{0}\right)=\left\{y_{1}, y_{2}, \ldots, y_{m}\right\}$ there exist special neighborhoods $\hat{V}_{i}\left(y_{i}\right)$. For each $\left(x_{0}, y_{i}\right)$ choose a product neighborhood $U_{i} \times V_{i} \subset B$ such that $V_{i} \subset \hat{V}_{i}$. Then by property (2a) there exists a neighborhood $U(x)$ such that

$$
\sum_{y \in V_{i}} \tilde{f}(x, y)=\widetilde{f}\left(x_{0}, y_{i}\right), \quad \text { all } x \in U .
$$

Putting $U^{\prime}=U \cap\left[\bigcap_{i} U_{i}\right]$ we have 


$$
\sum_{y \in V_{i}} \widetilde{f}_{1}(x, y)=\sum_{y \in V_{i}} \tilde{f}(x, y)=\widetilde{f}\left(x_{0}, y_{i}\right)=\widetilde{f}_{1}\left(x_{0}, y_{i}\right),
$$

for all $x \in U^{\prime}$.

Proposition 2.12. If $f: X \rightarrow Y$ is an m-function and $f_{1}: X \rightarrow Y$ is any component of $f$, then $f_{1}$ is an m-function.

Proof. Again $f_{1}$ clearly satisfies properties (1) and (2b). We consider a point $\left(x_{1}, y_{1}\right) \in f_{1}$ and prove (2a). Suppose that $U\left(x_{1}\right)$ and $V\left(y_{1}\right)$ satisfy (2a) for $f$; then $g^{\prime}=f \cap(U \times V)$ defines an $m$-function $g$ from $U$ into $Y$ of multiplicity $\widetilde{f}\left(x_{1}, y_{1}\right)$. Now we choose any $x \in U$ and show that

$$
\sum_{y \in V} \widetilde{f}_{1}(x, y)=\widetilde{f}_{1}\left(x_{1}, y_{1}\right)
$$

We know that

$$
\begin{aligned}
\sum_{y \in V} \tilde{f}(x, y)= & \sum_{y \in V ; y \in f_{1}^{\prime}(x)} \tilde{f}(x, y) \\
& +\sum_{y \in V ; y \notin f_{1}^{\prime}(x)} \tilde{f}(x, y)=\widetilde{f}\left(x_{1}, y_{1}\right)
\end{aligned}
$$

or

$$
\sum_{y \in V} \widetilde{f}_{1}(x, y)+\sum_{y \in V ; y \notin f_{1}^{\prime}(x)} \tilde{f}(x, y)=\widetilde{f}_{1}\left(x_{1}, y_{1}\right) .
$$

We need only show that the second sum is zero. It is finite, and each term of the sum arises from a point $(x, y)$ which is in some component of $f^{\prime}$; call these components $f_{2}^{\prime}, f_{3}^{\prime}, \ldots, f_{n}^{\prime}$. There is a separation $\left(B_{1}, B_{2}\right)$ of $f^{\prime}$ with $f_{1}^{\prime} \subset B_{1}$, $f_{2}^{\prime} \cup \cdots \cup f_{m}^{\prime} \subset B_{2}$. Now $g^{\prime} \cap B_{2}=g_{2}^{\prime}: U \rightarrow Y$ defines an $m$-function by Lemma 2.11 and its multiplicity is zero because $g_{2}^{\prime}\left(x_{1}\right)=\varnothing$.Therefore

$$
\sum_{y \in V: y \notin f_{1}^{\prime}(x)} \tilde{f}(x, y)=\sum_{y \in V} \tilde{g}_{2}(x, y)=0 .
$$

DEFinition 2.13. A full component of an $m$-function $f: X \rightarrow Y \times A$ is a component $m$-function $f_{1}$ such that for each $x \in X, f_{1}^{\prime}(x) \neq \varnothing$.

THEOREM 2.14. If $X$ is connected, an m-function $f: X \rightarrow Y \times A$ of nonzero multiplicity has a full component.

Proof. If a component $m$-function $f_{1}$ is not full there exists $x \in X$ such that $f_{1}^{\prime}(x)=\varnothing$ and therefore $m\left(f_{1}\right)=0$. Hence if $f$ has no full component, $m(f)=0$, a contradiction. 
By an abuse of language we shall call a component $m$-function $f_{1}$ of $f$ simply a component of $f$. This theorem shows that an $m$-function $f$ of nonzero multiplicity on a connected set has a component $f_{1}$ that is like a continuous function in that $f_{1}^{\prime} \subset X \times Y$ is connected and its projection covers $X$. It can be regarded as a selection theorem which singles out the full components.

We next define addition of $m$-functions and show that the $m$-functions over $A$ from $X$ to $Y$ form an $A$-module.

Definition 2.15. If $f, g: X \rightarrow Y \times A$ are $m$-functions, then $(f+g): X$ $\rightarrow Y \times A$ is defined by

$$
\tilde{f} \tilde{+} \tilde{g}(x, y)=\tilde{f}(x, y)+\tilde{g}(x, y) .
$$

This addition is clearly associative.

Proposition 2.16. Given m-functions $f, g: X \rightarrow Y \times A, f+g$ is an $m$ : function.

Proof. Property (1) is clearly satisfied. For property (2) we use the given neighborhoods for $\widetilde{f}$ and $\tilde{g}$. To make the $Y$-neighborhoods disjoint may require taking smaller neighborhoods, using the fact that $Y$ is Hausdorff. We use the intersection of the $X$-neighborhoods.

THEOREM 2.17. The $m$-functions from $X$ to $Y$ over $A$ form an A-module.

Proof. First, under the operation of addition the set of $m$-functions from $X$ to $Y$ forms an Abelian group. The zero $m$-function $0: X \rightarrow Y \times A$ is the empty $m$-function. Given $f,-f$ is defined by $\widetilde{-} \widetilde{f}(x, y)=-\widetilde{f}(x, y)$. The multiplication by elements of $A$ is given by $\widetilde{a} \tilde{f}(x, y)=a \widetilde{f}(x, y)$, so that distributive laws hold, $0 f=0$ and $1_{A} f=f$.

We note that the $m$-functions from $X$ to $X$ over $A$ form a semigroup under composition with identity $1_{X}$. They satisfy distributive laws. For example $f \circ$ $(g+h)$ is defined by

$$
\begin{aligned}
\sum_{x_{2} \in X}\left[\tilde{g}\left(x_{1}, x_{2}\right)+\tilde{h}\left(x_{1}, x_{2}\right)\right] \tilde{f}\left(x_{2}, x_{3}\right) \\
\quad=\sum_{x_{2} \in X} \tilde{g}\left(x_{1}, x_{2}\right) \tilde{f}\left(x_{2}, x_{3}\right)+\sum_{x_{2} \in X} \tilde{h}\left(x_{1}, x_{2}\right) \tilde{f}\left(x_{2}, x_{3}\right) \\
\quad=\tilde{f} \approx \tilde{g}\left(x_{1}, x_{3}\right)+\tilde{f} \approx \tilde{h}\left(x_{1}, x_{3}\right),
\end{aligned}
$$

the defining function of $f \circ g+f \circ h$.

We can now summarize the properties of $m$-functions.

THEOREM 2.18. (1) $m$-functions over $A$ and Hausdorff spaces form a category $M_{A}$. 
(2) Each m-function has a well-defined multiplicity.

(3) Each component of an m-function is an m-function.

(4) Each m-function of nonzero multiplicity has a full component.

(5) In $M_{A}, \operatorname{Hom}(X, Y)$ is an A-module.

The proofs of these facts are given above.

From this point on we shall denote an $m$-function $f: X \rightarrow Y \times A$ simply by $f: X \rightarrow Y$.

3. Applications to fixed points. In order to apply $m$-functions to fixed point theory we shall need to use an index for a fixed point of an ordinary function. We use Dold's fixed point index [2], defined axiomatically by Brown [1] as follows. A triple $(X, g, U)$ is admissible if $X$ is a connected space with $H^{*}(X, Q)$ finitary ( $Q$ = rationals), $g: X \rightarrow X$ is a continuous function, and $U \subset X$ is open with no fixed points of $g$ on $\operatorname{Bd}(U)$. The index is a function $i$ from the set of admissible triples to $Q$ satisfying these axioms:

Axioms 3.1. 1. If $g(x)=h(x)$ for all $x \in \operatorname{cl}(U)$, then $i(X, g, U)=$ $i(X, h, U)$.

2. If $f_{0}$ is homotopic to $f_{1}$ by a homotopy described by $f_{t}$, and if $(X$, $\left.f_{t}, U\right)$ is admissible for all $t \in[0,1]$, then $i\left(X, f_{0}, U\right)=i\left(X, f_{1}, U\right)$.

3. If $(X, f, U)$ is admissible and $\left\{U_{1}, U_{2}, \ldots, U_{s}\right\}$ is a set of mutually disjoint open subsets of $U$ such that $f(x) \neq x$ for all $x \in\left[U-\bigcup_{j=1}^{s} U_{j}\right]$, then $i(X, f, U)=\Sigma_{j=1}^{s} i\left(X, f, U_{j}\right)$.

4. $i(X, f, X)=L(f)$, The Lefschetz number of $f$ (with respect to rational cohomology).

5. If $f: X \rightarrow Y, g: Y \rightarrow X$ are such that $(X, g f, U)$ is admissible, then $i(X, g f, U)=i\left(Y, f g, g^{-1}(U)\right)$. Various spaces possess an index satisfying these axioms, compact ANR's among them. We shall call such spaces Dold index spaces.

The index axioms have some features in common with the $m$-function definition. The crux of this paper is to use this similarity to define an $m$-function arising from the fixed point properties of product spaces. We begin with a continuous function $f: X \times Y \rightarrow X \times Y$; we want conditions under which $f$ has a fixed point, or under which $X \times Y$ has the f.p.p. Using the projections $p_{1}: X \times Y \rightarrow X, p_{2}: X \times Y \rightarrow Y$ we define two families of functions:

$$
\begin{aligned}
f_{x}: Y & \rightarrow Y & f_{y}: X & \rightarrow X \\
y & \mapsto p_{2} f(x, y), & x & \mapsto p_{1} f(x, y) .
\end{aligned}
$$

We use these in turn to define certain multiple-valued functions.

DEFINITION 3.2. If $X$ and $Y$ are Dold index spaces, and if $f_{x}\left(f_{y}\right)$ has a finite number of fixed points for each $x \in X(y \in Y)$ we can define 


$$
\begin{array}{ll}
\phi: X \rightarrow Y & \psi: Y \rightarrow X \\
\tilde{\phi}(x, y)=i\left(Y, f_{x}, y\right), & \tau(y, x)=i\left(X, f_{y}, x\right) .
\end{array}
$$

Since the functions $f_{x}, f_{y}$ have a finite number of fixed points we can replace the open sets used in the axioms by isolated points.

The elements of $\phi^{\prime}(x)$ are essentially the fixed points of $f_{x}$, since $i\left(Y, f_{x}, Y\right)$ $\neq 0$ implies that $f_{x}(y)=y$. Actually in defining $\phi$ we discard any fixed points of $f_{x}$ of index zero which are not in the closure, in $X \times Y$, of the set of all fixed points of nonzero index. Where necessary we shall use a subscript $\phi_{f}$ to indicate the function from which $\phi$ is defined.

THEOREM 3.3. If $X$ and $Y$ are compact, connected, locally path-connected, Dold index spaces, and $f: X \times Y \rightarrow X \times Y$ is a continuous function such that for each $x$ and $y, f_{x}$ and $f_{y}$ have a finite number of fixed points, then $\phi$ and $\psi$ are $m$-functions over $Q$ with $m(\phi)=L\left(f_{x}\right), m(\psi)=L\left(f_{y}\right)$.

Proof FOR $\phi$. Note that $X$ and $Y$ are both path-connected, and therefore $L\left(f_{x}\right)$ and $L\left(f_{y}\right)$ are independent of $x$ and $y$. We first show that $\phi^{\prime}(x)$ is a finite set. If $y \in \phi^{\prime}(x)$ either $y$ is a fixed point of $f_{x}$ of nonzero index, or it is a cluster point in $X \times Y$ of such points. But the set $\left\{(s, t) \mid p_{2} f(s, t)=t\right\}$ is closed in $X \times Y$, so in either case $y$ is a fixed point of $f_{x}$. The number of such points is finite by hypothesis.

We can now assume that, for $x_{0} \in X, \phi^{\prime}\left(x_{0}\right)=\left\{y_{1}, y_{2}, \ldots, y_{n}\right\}$. We choose neighborhoods $\hat{V}_{j}\left(y_{j}\right)$ such that their (compact) closures are pairwise disjoint. Then, again by compactness, there exists a neighborhood $U^{\prime}\left(x_{0}\right)$ such that $f_{x}$ has no fixed points on $\operatorname{Bd}\left(\hat{V}_{j}\right)$ for any $x \in U^{\prime}(j=1,2, \ldots, n)$. Since $X$ is locally path-connected there exists a neighborhood $U\left(x_{0}\right) \subset U^{\prime}$ such that any two points in $U$ are connected by a path in $U^{\prime}$. We choose any $x_{1} \in U$ and a path $\alpha: I \rightarrow U^{\prime}$ from $x_{0}$ to $x_{1}$. Then $f_{\alpha(t)}$ describes a homotopy from $f_{x_{0}}$ to $f_{x_{1}}$ and by Axiom 2,

$$
i\left(Y, f_{x_{0}}, \hat{V}_{j}\right)=i\left(Y, f_{x_{1}}, \hat{V}_{j}\right)=\tilde{\phi}\left(x_{0}, y_{j}\right) .
$$

Now suppose that $\phi^{\prime}\left(x_{1}\right) \cap \hat{V}_{j}=\left\{y_{j 1}, y_{j 2}, \ldots, y_{j m_{j}}\right\}$. We choose mutually disjoint neighborhoods $V_{j k}\left(y_{j k}\right) \subset \hat{V}_{j}$, and by Axiom 3,

or

$$
i\left(Y, f_{x_{1}}, \hat{V}_{j}\right)=\sum_{k=1}^{m_{j}} i\left(Y, f_{x_{1}}, V_{j k}\right)
$$

$$
\widetilde{\phi}\left(x_{0}, y_{j}\right)=\sum_{k=1}^{m_{j}} \widetilde{\phi}\left(x_{1}, y_{j k}\right)=\sum_{y \in \hat{V}_{j}} \widetilde{\phi}\left(x_{1}, y\right) .
$$


Since this is true for any $x_{1} \in U$ the proof of property (2a) in the $m$-function definition is complete. Property (2b) follows from the compactness of $Y(2.4)$, so $\phi$ is an $m$-function.

It follows immediately from Axioms 3 and 4 that the Lefschetz number

$$
\begin{aligned}
L\left(f_{x}\right) & =i\left(Y, f_{x_{0}}, Y\right)=\sum_{k=1}^{n} i\left(y, f_{x_{0}}, y_{k}\right) \\
& =\sum_{k=1}^{n} \tilde{\phi}\left(x_{0}, y_{k}\right)=\sum_{y \in Y} \tilde{\phi}\left(x_{0}, y\right)=m(\phi) .
\end{aligned}
$$

The proof for $\psi$ is the same.

We note that if $F: I \times X \rightarrow X$ is a homotopy this theorem applies. We convert it to a level-preserving homotopy $G: I \times X \rightarrow I \times X[G(t, x)=$ $(t, F(t, x))]$ and then if the conditions of the theorem are satisfied the fixed points of $F_{t}$, indexed by $t$, define an $m$-function $\phi_{F}: I \rightarrow X$. If $L\left(F_{t}\right) \neq 0, \phi_{F}$ will have a full component.

Returning to $\phi_{f}$ and $\psi_{f}$, if their graphs intersect in $X \times Y$ the point of intersection is a fixed point of $f$. To examine this situation we consider the compositions of these functions.

Definitions. The $m$-functions over $Q$

$$
\begin{aligned}
\xi_{f} & =\psi \circ \phi: X \rightarrow X, & \eta_{f} & =\phi \circ \psi: Y \rightarrow Y, \\
\tilde{\xi}_{f}\left(x, x^{\prime}\right) & =\sum_{y \in Y} i\left(Y, f_{x}, y\right) i\left(X, f_{y}, x^{\prime}\right), & \tilde{\eta}_{f}\left(y, y^{\prime}\right) & =\sum_{x \in X} i\left(X, f_{y}, x\right) i\left(Y, f_{x}, y^{\prime}\right)
\end{aligned}
$$

have multiplicities

$$
m(\xi)=m(\psi) m(\phi)=L\left(f_{y}\right) L\left(f_{x}\right), \quad m(\eta)=m(\phi) m(\psi)=L\left(f_{x}\right) L\left(f_{y}\right) .
$$

We shall drop the subscripts on $\xi, \eta$ except where necessary. Because of the symmetry in this situation we will deal only with $\xi$. Equivalent results are obtained by using $\eta$. We first obtain a connection between $\xi$ and the Lefschetz number of $f$.

Proposition 3.4. $\Sigma_{x \in X} \tilde{\xi}(x, x)=\Sigma_{y \in Y} \tilde{\eta}(y, y)=L(f)$.

Proof. We have

$$
\sum_{x \in X} \tilde{\xi}(x, x)=\sum_{x \in X} \sum_{y \in Y} i\left(Y, f_{x}, y\right) i\left(X, f_{y}, x\right)
$$

and use the fact (Brown [1]) that

$$
i\left(Y, f_{x}, y\right) i\left(X, f_{y}, x\right)=i\left(X \times Y, f_{y} \times f_{x},(x, y)\right) .
$$

Then 


$$
\sum_{x \in X} \tilde{\xi}(x, x)=\sum_{(x, y) \in X \times Y} i(X \times Y, f,(x, y))=i(X \times Y, f, X \times Y)=L(f)
$$

We say that the $m$-function $\xi$ has a fixed point if there exists $x \in X$ such that $x \in \xi^{\prime}(x)$, that is, if $\xi^{\prime}$ has a fixed point.

LEмma 3.5. In the situation of Theorem 3.3, if $\xi$ has a fixed point $a \in X$, then $f$ has a fixed point $(a, y)$ for some $y \in Y$.

Proof. Suppose that $a \in \xi(a)$. Then

$$
(a, a) \in \operatorname{cl}\left\{\left(x, x^{\prime}\right) \mid \tilde{\xi}\left(x, x^{\prime}\right) \neq 0\right\} .
$$

If $f$ has no fixed point $(a, y)$, then by compactness there is a neighborhood $W(a)$ such that if $(x, y) \in W \times Y$ then either $f_{x}(y) \neq y$ or $f_{y}(x) \neq x$. Hence either $i\left(X, f_{y}, x\right)=0$ or $i\left(Y, f_{x}, y\right)=0$, and if $\left(x, x^{\prime}\right) \in W \times W$,

$$
\tilde{\xi}\left(x, x^{\prime}\right)=\sum_{y \in Y} i\left(Y, f_{x}, y\right) i\left(X, f_{y}, x^{\prime}\right)=0 .
$$

This is a contradiction.

The question of whether $f$ has a fixed point in $X \times Y$ is now reduced to the question of whether the associated $m$-function $\xi_{f}$ has a fixed point in $X$. We say that $X$ has the $m$-fixed point property (abbreviated $m$-f.p.p.) if each $m$-function of nonzero multiplicity on $X$ has a fixed point. The restriction to $m$-functions of nonzero multiplicity is necessary because $m$-functions of multiplicity zero include the empty $m$-function whose defining function maps $X \times Y$ onto zero. The restriction also makes sense in the light of Theorem 2.14. We are now ready to state our main theorem on fixed points in product spaces.

THEOREM 3.6. Given continuous $f: X \times Y \rightarrow X \times Y$, satisfying

(a) $X$ and $Y$ are connected, compact, locally path-connected Dold index spaces,

(b) $f_{x}$ and $f_{y}$ each have only a finite number of fixed points for each $x \in$ $X, y \in Y$,

(c) the $m$-function $\xi_{f}: X \rightarrow X$ associated with $f$ has a fixed point, then $f$ has a fixed point.

Proof. Conditions (a) and (b) insure that $\xi$ (and $\eta$ ) are well defined and are $m$-functions. Condition (c) and Lemma 3.5 give the result.

COROllaRY 3.7. In Theorem 3.6 we replace condition (c) by the stronger conditions

(c $\left.c_{1}\right) L\left(f_{x}\right) \neq 0, L\left(f_{y}\right) \neq 0$, and

( $\left.c_{2}\right) X$ or $Y$ has the m-f.p.p. 
Proof. Condition $\left(c_{1}\right)$ assures that $m(\xi)=L\left(f_{x}\right) L\left(f_{y}\right) \neq 0$, as required for the $m$-f.p.p.

These results reduce the proof of the existence of a fixed point of $f$ to the proof of the existence of a fixed point of $\xi_{f}$. Roughly, the question of the f.p.p. for a product space is reduced to the question of the $m$-f.p.p. for one of the factor spaces. Considering Theorem 2.14 it is reasonable to suppose that a fairly wide class of spaces has the $m$-f.p.p.

There are various ways of applying the theorem. One possibility is to generalize the definition of $m$-functions to remove the unpleasant condition (b). However, in the following sections we shall prove a Lefschetz fixed point theorem for $m$-functions on polyhedra to illuminate condition (c), and satisfy condition (b) by approximating $f$. For the Lefschetz theorem we shall need homology with $m$ functions, which we take up in the next section.

4. $m$-homology. The category $M_{A}$ of $m$-functions over a $\operatorname{ring} A$ and Hausdorff spaces seems a natural one on which to define a singular $m$-homology functor. The whole apparatus is defined with very little change from the usual definition, the proofs of the axioms being formally the same as the proofs for ordinary singular homology. By uniqueness, the $m$-homology modules of polyhedra are isomorphic to the ordinary homology modules, but $m$-functions will induce homomorphisms of the homology modules which may not appear in the usual theory. The relationship between the two theories is not clear.

We start with $\Delta^{n}$, the standard geometric $n$-simplex in $E^{n+1}$, and the standard boundary maps $\partial_{i}: \Delta^{n-1} \rightarrow \Delta^{n}(i=1,2, \ldots, n+1)$, which will be regarded as $m$-functions.

Definitions 4.1. (a) An $n$-simplex in a Hausdorff space $X$ is an $m$-function $\sigma^{n}: \Delta^{n} \rightarrow X$ (over the ring $A$ ).

(b) $C_{n}(X)=\operatorname{Hom}\left(\Delta^{n}, X\right)$ is the $A$-module of all $n$-simplices in $X$ if $n \geqslant 0$; it is zero if $n<0$.

(c) The boundary map $\partial: C_{n}(X) \rightarrow C_{n-1}(X)$ is defined by

$$
\partial \sigma^{n}=\sum_{i=1}^{n+1}(-1)^{i} \sigma^{n} \circ \partial_{i} .
$$

The proof that $\partial \partial=0$ is exactly the same as for ordinary simplicial homology. Thus $\left\{C_{n}(X), \partial\right\}$ is a chain complex. We put $Z_{n}(C)=\operatorname{ker} \partial, B_{n}(C)=\operatorname{Im} \partial$ and define $H_{n}(X)=Z_{n}(C) / B_{n}(C)$ to be the $n$th $m$-homology module of $X$ over $A$.

THEOREM 4.2. m-homology over a ring $A$ is a functor from $M_{A}$ to the category of graded A-modules.

Proof. We have assigned to each Hausdorff space $X$ the graded $A$-module 
$H_{*}(X)$. If $Y$ is another Hausdorff space we have chain complexes $\left\{C_{n}(X), \partial\right\}$ and $\left\{C_{n}(Y), \partial\right\}$, and if $f: X \rightarrow Y$ is an $m$-function it induces a chain map $\hat{f}: C_{n}(X)$ $\rightarrow C_{n}(Y)$ in the usual way: if $\sigma$ is a simplex in $X$ then $f \sigma$ is a simplex in $Y$ and we define

$$
\begin{gathered}
C_{n}(X) \stackrel{\hat{f}}{\rightarrow} C_{n}(Y) \\
\sigma^{n} \mapsto f \sigma^{n} .
\end{gathered}
$$

Each element of the chain $C_{n}(X)$, which would in the usual theory be a linear combination of ordinary singular simplices, is a single $m$-homology simplex. The formation of the chain $A$-modules has been subsumed into the definition of the $m$-function $A$-modules. We find that $\hat{f} \partial=\partial \hat{f}$ and that $1_{X}$ (regarded as an $m$ function) induces the identity map on $C_{n}(X)$. Further, if $g: Y \rightarrow Z$ is an $m$ function, then clearly $\widehat{g} f=\hat{g} \hat{f}$. Therefore we have a functor from $M_{A}$ to chain complexes and chain maps. We compose this with the usual functor to graded $A$-modules to get the required functor. An $m$-function $f: X \rightarrow Y$ induces an $A$ module homomorphism $f_{*}: H_{*}(X) \rightarrow H_{*}(Y)$.

THEOREM 4.3. m-homology is a homology theory.

Proof. We must prove the Eilenberg-Steenrod Axioms

(a) m-homotopy invariance.

DEFinition. If $f, g: X \rightarrow Y$ are $m$-functions, then $f$ is $m$-homotopic to $g(f \sim g)$ if there exists an $m$-function $F: X \times I \rightarrow Y$ such that $F(x, 0)=f(x)$, $F(x, 1)=g(x)$. We claim that if $f \sim g$ then $f_{*}=g_{*}$. To prove this we define

$$
\begin{aligned}
\lambda_{t}: X & \rightarrow X \times I \\
x & \rightarrow(x, t)
\end{aligned}
$$

and consider $X \stackrel{\lambda_{t}}{\rightarrow} X \times I \stackrel{F}{\rightarrow} Y$. We have $f=F \lambda_{0}, g=F \lambda_{1}$. If the claim is true for $\lambda_{0} \sim \lambda_{1}$ (i.e. $\lambda_{0_{*}}=\lambda_{1 *}$ ) then it is true for $f \sim g$, for

$$
f_{*}=\left(F \lambda_{0}\right)_{*}=F_{*} \lambda_{0 *}=F_{*} \lambda_{1 *}=\left(F \lambda_{1}\right)_{*}=g_{*} .
$$

But the proof that $\lambda_{0 *}=\lambda_{1 *}$ does not involve $m$-functions.

(b) Exact $m$-homology sequence. If $B \subset X$ we define $C_{n}(B)$ to be the $A$ module of $m$-homology simplices $\sigma^{n}: \Delta^{n} \rightarrow B$, and we define $\bar{\partial}$ in the usual way so that the diagram

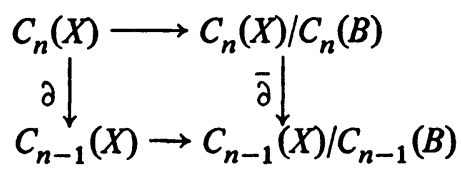


commutes. The relative $m$-homology modules $H_{n}(X, B)$ are defined as before, and the usual proof of the exact sequence theorem is not affected by the introduction of $m$-functions.

(c) Excision theorem. As in ordinary singular homology we construct a subdivision operator Sd: $C_{n}(X) \rightarrow C_{n}(X)$ and a "roof" operator $R: C_{n}(X) \rightarrow$ $C_{n+1}(X)$. Denoting the identity on $\Delta^{n}$ by $1_{n}$ we define $\operatorname{Sd}\left(1_{n}\right)$ inductively by the usual formulas

$$
\operatorname{Sd}\left(1_{0}\right)=1_{0}, \quad \operatorname{Sd}\left(1_{n}\right)=b * \operatorname{Sd}\left(\partial 1_{n}\right)
$$

where $b * \operatorname{Sd}\left(\partial 1_{n}\right)$ represents the sum of those simplices which map $\Delta^{n}$ affinely onto the joins of the barycenter of $\Delta^{n}$ with the images of the simplices in $\operatorname{Sd}\left(\partial 1_{n}\right)$. Since we are regarding all simplices as $m$-functions, this sum is again an $m$-function and therefore an $n$-simplex. For $\sigma \in C_{n}(x)$ we have $\operatorname{Sd}(\sigma)=$ $\sigma \circ\left(\operatorname{Sd}\left(1_{n}\right)\right)$, which is an $n$-simplex expressed as a sum of $n$-simplices with smaller images. The operator $R$ is also defined by the usual formulas.

It then follows, using $S d$ and $R$ as in the usual proof, that if $\sigma \in H_{n}(X, B)$ is a relative $n$-cycle (which can also be regarded as an $n$-simplex) then $\sigma$ is homologous to $\mathrm{Sd}^{r} \sigma(\bmod B)$.

Given any open cover $V=\left\{V_{i}\right\}$ of $X$ and an $n$-simplex $\sigma: \Delta^{n} \rightarrow X$, the $m$-function Definition 2.1 shows that if $y \in \Delta^{n}$ there is a neighborhood $U_{y}$ of $y$ such that the image of $U_{y}$ under $\sigma^{\prime}$ is contained in a finite union of disjoint elements of $V$. Since $\Delta^{n}$ is compact there is an open cover $U=\left\{U_{i}\right\}$ of $\Delta^{n}$ such that each element of $U$ is mapped into a finite union of disjoint elements of $V$. By 2.12, $\sigma \mid U_{j}$ is a finite union of $m$-functions, each of which maps $U_{j}$ into an element of $V$. We choose $r$ so that $\operatorname{Sd}^{r}\left(1_{n}\right)=\Sigma \alpha_{i}\left(\alpha_{i}: \Delta^{n} \rightarrow \Delta^{n}\right)$ is a finite sum of simplices each of whose images in $\Delta^{n}$ lies in some element of $U$. Then

$$
\operatorname{Sd}^{r}(\sigma)=\sigma \circ \operatorname{Sd}^{r}\left(1_{n}\right)=\sigma \circ \sum \alpha_{i}=\sum \sigma \circ \alpha_{i},
$$

and every homology class in $H_{n}(X, B)$ can be represented by a relative cycle which is a sum of $n$-simplices each of which has its image in $X$ in an element of $V$. With this key fact the rest of the proof is conventional.

(d) Dimension axiom. We compute the $m$-homology modules of a point. The only simplices $\sigma^{n}: \Delta^{n} \rightarrow p$ are given by $\widetilde{\sigma}^{n}(x, p)=a \in A$, and therefore $C_{n}(p)=A$. From this point the calculation is as usual, and $H_{0}(p)=A, H_{n}(p)$ $=0$ if $n>0$. This concludes the proof of the axioms, and therefore $m$-homology is a homology theory.

We do not have any very satisfactory set of generators for the $A$-module $\operatorname{Hom}(X, Y)$. One set of generators is the connected component $m$-functions of multiplicity either one or zero (see 2.12). If $\operatorname{Hom}(X, Y)$ is generated by the continuous functions, then $m$-homology is essentially the same as ordinary ho- 
mology. One can also define $m$-cohomology, $m$-fundamental group, etc. It seems possible to replace functions by $m$-functions in the whole apparatus of algebraic topology. However, in this paper we do not take up any aspect of $m$-homology except what is needed to prove a Lefschetz theorem.

5. Piecewise linear $m$-functions; Lefschetz theorem. Due to the fact that $m$-functions are multiple-valued, the construction of simplicial $m$-functions is slightly complicated. We start with the definitions.

Definition 5.1. An $m$-function $f: X \rightarrow Y$ is piecewise linear if $f^{\prime} \subset X \times$ $Y$ is a polyhedron. If $K, L$ are triangulations of $X, Y$, then $f: K \rightarrow L$ is simplicial if it is a piecewise linear $m$-function such that for each $\sigma \in K, f^{\prime}(\sigma)$ is a finite collection of simplices of $L,\left\{\tau_{1}, \tau_{2}, \ldots, \tau_{n}\right\}$ with each map $\sigma \rightarrow \tau_{i}$ affine.

If $f: X \rightarrow Y$ and $g: Y \rightarrow Z$ are piecewise linear $m$-functions it follows from the usual proof for functions that $g^{\prime} \circ f^{\prime}: X \rightarrow Z$ is a multiple-valued function whose graph is a polyhedron in $X \times Z$. As subsets of $X \times Z,(g \circ f)^{\prime}$ $\subset g^{\prime} \circ f^{\prime}$. To get $(g \circ f)^{\prime}$ we can assign a multiplicity to each point in $g^{\prime} \circ f^{\prime}$ by the rule for compositon of $m$-functions, and then take the closure of the set of points in $g^{\prime} \circ f^{\prime}$ which have nonzero multiplicity. Because $f$ and $g$ are piecewise linear, the multiplicity will be zero on a closed subpolyhedron of $g^{\prime} \circ f^{\prime}$. Therefore $(g \circ f) \subset X \times Z$ is a polyhedron and the composition of piecewise linear $m$-functions is piecewise linear.

THEOREM 5.2. Given a piecewise linear m-function $f: X \rightarrow Y$ and triangulations $K, L$ of $X, Y$ there exist subdivisions $K_{1}, L_{1}$ of $K, L$ and an $m$-function $f_{1}: K_{1} \rightarrow L_{1}$ that is a simplicial approximation to $f ; f_{1}$ approximates $f$ to within the mesh of $L_{1}$.

Proof. We denote by $\Gamma$ the graph of $f^{\prime}$ and by $G$ a cellular subdivision of $\Gamma$, which exists because $\Gamma$ is a polyhedron. There exist triangulations $G_{1}$ and $L_{1}$, subdivisions of $G$ and $L$, such that the projection $p_{2}: G_{1} \rightarrow L_{1}$ is simplicial. Since $f^{\prime}$ is multiple-valued, the projection $p_{1}: G_{1} \rightarrow X$ induces a cellular subdivision of $X$. We take a triangulation $K_{1}$ of $X$ that is a common subdivision of $K$ and of this cellular subdivision.

We next construct a simplicial $m$-function approximating $f$ as follows. If $v$ is a vertex of $K_{1}, f(v)=\left\{p_{1}, p_{2}, \ldots, p_{n}\right\}$, where $p_{i} \in Y$. If $p_{i}$ is a vertex $w_{i}$ of $L_{1}$ we make no change. If $p_{j}$ is not a vertex of $L_{1}$ then $\left(v, p_{j}\right) \in \mu \in G_{1}$, and $p_{2}(\mu)=\tau \in L_{1}$. We redefine the $j$ th image of $v$ to be $w_{j}$, where $w_{j}$ is the vertex of $\tau$ nearest to $p_{j}$. This defines a simplicial $m$-function $f_{1}: K_{1} \rightarrow L_{1}$ by linear extension over each simplex of $K_{1}$. It amounts to altering $f^{\prime}$ to $f_{1}^{\prime}$ by a small homotopy. The multiplicity assigned to each point is unchanged, except that when two or more image points of $f^{\prime}(x)$ coincide under $f_{1}^{\prime}$, the multiplicity 
of the new image point is the sum of the multiplicities of the formerly separate points which it represents.

We note that the projection $p_{1}$ and the triangulation $K_{1}$ of $X$ induce a triangulation $G_{2}$ of $\Gamma$ so that $p_{1}: G_{2} \rightarrow K_{1}$ is simplicial. If $\sigma \in K_{1}$ then $p_{1}^{-1}(\sigma)$ $\cap \Gamma=\left\{\gamma_{1}, \gamma_{2}, \ldots, \gamma_{s}\right\}$ where $\gamma_{i} \in G_{2}, \gamma_{i} \subset \mu_{i} \in G_{1}$, and $p_{2}\left(\mu_{i}\right) \in \tau_{i} \in L_{1}$. In replacing $f^{\prime}$ by $f_{1}^{\prime}$ we replace each $\gamma_{i}$ by a simplex $\gamma_{i}^{\prime}$; this is done by moving the $Y$-coordinates of its vertices within $\tau_{i}$ to the vertices of $\tau_{i}$. Then $p_{1}\left(\gamma_{i}^{\prime}\right)=\sigma$ and $p_{2}\left(\gamma_{i}^{\prime}\right)$ is a face of $\tau_{i}$. The graph $\Gamma^{\prime}$ of $f_{1}^{\prime}$ is triangulated by the set of altered simplices, $\left|G^{\prime}\right|=\Gamma^{\prime}$, and $p_{1}: G^{\prime} \rightarrow K_{1}, p_{2}: G^{\prime} \rightarrow L_{1}$ and $f_{1}^{\prime}: K_{1} \rightarrow L_{1}$ are all simplicial.

COROLlaRY 5.3. Given a piecewise linear m-function $f: X \rightarrow X$ and a triangulation $K$ of $X$ there exists a subdivision $K^{\prime}$ of $K$, an rth derived subdivision $K_{r}^{\prime}$ of $K^{\prime}$, and an $m$-function $f_{1}: K_{r}^{\prime} \rightarrow K^{\prime}$ that is a simplicial approximation to $f_{\text {. }}$

Proof. As in the theorem above we take triangulations $K^{\prime}$ of $X$ (playing the role of $L_{1}$ ) and $G_{1}$ of $\Gamma$ such that $p_{2}: G_{1} \rightarrow K^{\prime}$ is simplicial. We take $K_{r}^{\prime}$ to be a common subdivision of $K^{\prime}$ and $p_{1}\left(G_{1}\right)$ and also a derived subdivision of $K^{\prime}$. Then we define, as above, $f_{1}: K_{r}^{\prime} \rightarrow K^{\prime}$, a simplicial $m$-function approximating $f$.

We do not have a simplicial approximation theorem for $m$-functions; for our purpose here it is sufficient to consider simplicial approximations of piecewise linear $m$-functions.

If $X$ is any space such that the graded $m$-homology $A$-module $H_{*}(X)$ is finitary, and if $f: X \rightarrow X$ is an $m$-function, then the Lefschetz number of $f$ is the Lefschetz number $L(f)$ of the induced homomorphism $f_{*}: H_{*}(X) \rightarrow H_{*}(X)$. In particular, if $X$ is a compact polyhedron, then by uniqueness the $m$-homology module over the integers is isomorphic to the usual homology module, so the Lefschetz number can be defined.

THEOREM 5.4 (Lefschetz' Theorem). If $X$ is a compact, connected polyhedron, $f: X \rightarrow X$ is a piecewise linear $m$-function over the integers and $L(f) \neq$ 0 , then $f$ has a fixed point.

Proof. By 5.3 there exists an arbitrarily close simplicial approximating $m$-function $F: K_{r} \rightarrow K$ where $K$ is a triangulation of $X$. If $F$ has a fixed point, then $f$ has a fixed point; also $L(f)=L(F)$. We assume that $F$ has no fixed point and that $K$ has been chosen so that $\sigma \cap F^{\prime}(\sigma)=\varnothing$ for each $\sigma \in K$. The proof is now conventional.

Let $\tau: C(K) \rightarrow C\left(K_{r}\right)$ be a subdivision chain map. We have

$$
C(K) \stackrel{\tau}{\rightarrow} C\left(K_{r}\right) \stackrel{C(F)}{\longrightarrow} C(K),
$$


single point or is a cell $c^{p}$ properly embedded in $\mu$. To prove this we note that since $p_{2} F$ is simplicial, we can assume that $p_{2} F\left(\mu^{k}\right)=\tau^{l} \in L$. By linearity the intersection of $\mu$ with the line joining any two points of $\alpha \cap \mu$ is in $\alpha \cap \mu$. Hence if there is more than one point of $\alpha$ in $\mu$ the set $\alpha \cap \mu$ is a linear subspace of $\mu$, i.e. a properly embedded cell of some dimension $p \geqslant 1$.

We next show that a suitable rotation of $c^{p}$ will insure that the rotated cell projects $1: 1$ into $X$. Choose $r$ so that each simplex $\mu \in M_{r}$ has smaller diameter than the smallest $\tau \in L$. Consider $\mu^{m} \in M_{r}, \mu^{m} \subset \nu^{n} \in M$, and suppose that the set $\left\{z \in \mu^{m} \mid p_{2} z=p_{2} F z\right\}$ is a properly embedded cell $c^{p} \subset \mu^{m}$. Put $p_{1} \nu^{n}=\kappa^{k}$ $\in K, p_{2} \nu^{n}=\lambda^{l} \in L$, and $p_{2} F\left(\mu^{m}\right)=\lambda_{1}^{l_{1}} \in L$; we claim that $p \leqslant k$. First we note that $m \leqslant n$ and $n \leqslant k+l$. The map $p_{2} F$ expands the simplex $\mu^{m}$ in $l_{1}$ orthogonal directions, but the cell $c^{p} \subset \mu^{m}$ is not expanded; hence $p \leqslant m-l_{1}$. Further, $p_{2} c^{p}=p_{2} F c^{p}$ implies that $\lambda^{l} \cap \lambda_{1}^{l_{1}}=\varnothing$, so either (a) $\lambda^{l} \leqslant \lambda_{1}^{l_{1}}$, or (b) $\lambda_{1}^{l_{1}}$ $<\lambda^{l}$. In case (a) we have $l \leqslant l_{1}$ and $m \leqslant n \leqslant k+l \leqslant k+l_{1}, p \leqslant m-l_{1} \leqslant k+$ $l_{1}-l_{1}=k$. In case (b) $\lambda_{1}^{l_{1}}$ is a proper face of $\lambda^{l}$. But since $c^{p} \subset \operatorname{int}\left(\mu^{m}\right) \subset$ $\operatorname{int}\left(v^{n}\right)$ it follows that $p_{2} c^{p} \subset \operatorname{int}\left(\lambda^{l}\right)$ and $p_{2} c^{p} \cap \lambda_{1}^{l_{1}}=\varnothing$ and therefore $c^{p}=\varnothing$. Then $p \leqslant k$ in both cases and the conclusion follows.

We now alter $M_{r}$ so that this is the case. The simplex $\mu$ has $k+1$ vertices, of which at least $k$ are starring points of simplices in $M_{r-1}$, and these can be varied in their respective simplices. Suppose that $v$ is a vertex of $\mu \in M_{r}$, the starring point of $\sigma \in M_{r-1}$. We can write $\mu=v \cdot B$ (join) where $B$ is a closed face of $\mu$. Altering the position of $v$ within $\sigma$ (and hence $M_{r}$ and the definition of $F$ ) will have no effect upon $c^{p} \cap B$, but motion of $v$ in almost any direction will change the $X$-coordinates of $c^{p} \cap(\mathrm{Bd} \mu-B)$ and will therefore rotate $c^{p}$. There are thus uncountably many ways of altering the starring vertices of $\mu$ so that $c^{p}$ projects $1: 1$ into $X$. It follows that by choosing $M_{r}$ properly we can insure that for each $x \in X$ the set $\alpha \cap p_{1}^{-1}(x)$ contains at most one element in each simplex of $M_{r}$ and is therefore finite. The proof for $\beta$ is the same, and since $\alpha$ and $\beta$ are both finite unions of cells, they are polyhedra. This completes the proof.

Given $F: X \times Y \rightarrow X \times Y$ satisfying 6.1 we can define $\phi_{F}$ and $\psi_{F}$ as in 3.2 and we note that any connected, compact polyhedron is a locally path-connected Dold index space. Then by Theorem 3.3 both $\phi: X \rightarrow Y$ and $\psi: Y \rightarrow X$ are $m$-functions. Since the fixed point index on such spaces is an integer, they are $m$-functions over the integers. Their composition $\xi_{F}: X \rightarrow X$ is a piecewise linear $m$-function. Now if $f: X \times Y \rightarrow X \times Y$ is given we define $L_{f}$ to be $\lim \left(L\left(\xi_{F}\right)\right.$ ), (when the limit makes sense) as the simplicial approximations $F$ converge to $f$. We can now state the main applications of Theorem 3.6.

TheOREM 6.2. If $X$ and $Y$ are compact, connected polyhedra, $f: X \times Y$ $\rightarrow X \times Y$ is a continuous function and $L_{f} \neq 0$, then $f$ has a fixed point. 
and since $C(F) \tau(\sigma)$ is a chain with coefficient zero on $\sigma, L(C(F) \tau)=0$. From the diagram

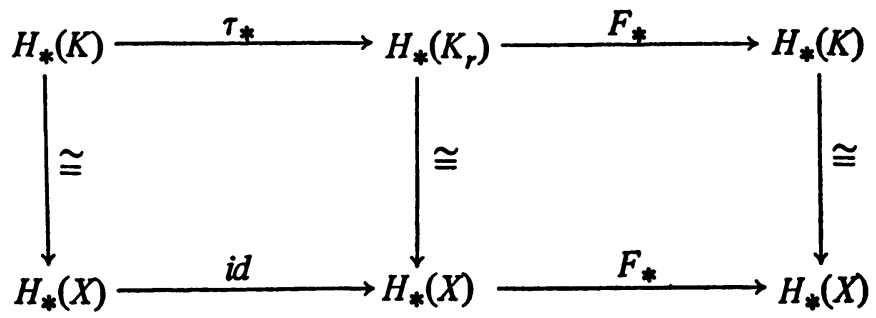

we have

$$
L(F)=L\left(F_{*}\right)=L\left(F_{*} \tau_{*}\right)=L(C(F) \tau)=0,
$$

a contradiction.

6. Applications to polyhedra. We now assume that $X$ and $Y$ are compact, connected polyhedra; we want to apply Theorem 3.6. We are given a continuous function $f: X \times Y \rightarrow X \times Y$ and will obtain a simplicial approximation $F$ to $f$ that satisfies condition (b) of 3.6. We put

$$
\alpha=\left\{(x, y) \mid F_{x}(y)=y\right\}, \quad \beta=\left\{(x, y) \mid F_{y}(x)=x\right\} .
$$

Recall now that $\phi_{F}: X \rightarrow Y$ is defined by $i\left(Y, F_{x}, y\right)$, and that the fixed point index for an isolated fixed point is the intersection number of the diagonal of $Y \times Y$ with the graph of $F_{x}$. Since $F$ is a piecewise linear, the subset of $\alpha$ on which this number is zero is a closed subpolyhedron $\alpha_{0} \subset \alpha$. The graph of $\phi^{\prime}$ is $\overline{\alpha-\alpha_{0}}$ and is also a polyhedron. Therefore, if $\alpha$ is a polyhedral subset of $X \times Y$, then $\phi_{F}$ is a piecewise linear; a similar conclusion holds for $\beta$ and $\Psi_{F}$.

LEMMA 6.1. Given compact, connected polyhedra $X, Y$ and continuous $f$ : $X \times Y \rightarrow X \times Y$ there exists an arbitrarily close simplicial approximation $F$ such that

(a) for each $x \in X$ and $y \in Y, F_{x}$ and $F_{y}$ each have a finite number of fixed points;

(b) $\alpha$ and $\beta$ are polyhedral subsets of $X \times Y$.

Proof. We take arbitrary triangulations $K, L$ of $X, Y, K \times L$ is a cellular subdivision of $X \times Y$ which we triangulate without adding vertices to obtain a triangulation $M$ of $X \times Y$. Denoting by $M_{r}$ an $r$ th derived subdivision of $M$, for some $r>0$ there exists a simplical approximation $F: M_{r} \rightarrow M$ to $f$. The projection $p_{1}: M \rightarrow K, p_{2}: M \rightarrow L$ are simplicial, and therefore the maps $p_{1} F, p_{2} F$ are simplicial. Note that $F_{x}=p_{2} F(x,-), F_{y}=p_{1} F(-, y)$ so, for example, $\alpha$ is the set of points whose $Y$-coordinate is unchanged by $p_{2} F$.

We first claim that in any (open) $\mu^{k} \in M_{r}, \alpha \cap \mu$ is either empty or is a 
Proof. We approximate $f$ by $F$ as in 6.1 , and need only show that $F$ has a fixed point. Conditions (a) and (b) of 3.6 are satisfied by $X, Y$ and $F$. Also, $L_{f} \neq 0$ implies $L\left(\xi_{F}\right) \neq 0$ and then by Theorem $5.4 \xi_{F}$ has a fixed point. Theorem 3.6 then shows that $F$ has a fixed point.

COROLlaRY 6.3. In Theorem 6.2 we can replace the condition $L_{f} \neq 0$, as in 3.7, with the conditions: $L\left(f_{x}\right) \neq 0, L\left(f_{y}\right) \neq 0$, and $X$ or $Y$ has the m-f.p.p.

We say that $X$ has property $F$ if for any continuous function $f: X \rightarrow X$, $L(f) \neq 0$. Further, $X$ has property $m-F$ if for each $m$-function $f: X \rightarrow X$ of nonzero multiplicity $L(f) \neq 0$. Clearly property $m \cdot F$ implies property $F$; we do not know if they are equivalent. Then from 6.3 we obtain the following.

Corollary 6.4. If $X, Y$ are compact, connected polyhedra, $X$ has property $F$ and $Y$ has property $m-F$, then $X \times Y$ has the f.p.p.

Corollary 6.5. If $X, Y$ are compact, connected polyhedra, $X$ has property $F$ and $Y$ is acyclic, then $Y$ has the m-f.p.p. and $X \times Y$ has the f.p.p.

Proof. Since $Y$ is acyclic, if $\eta: Y \rightarrow Y$ is an $m$-function of multiplicity $m \neq 0$, then $L(\eta)=m$.

\section{REFERENCES}

1. R. F. Brown, The Lefschetz fixed point theorem, Scott Foresman, Glenview Ill., 1971. MR 44 \#1023.

2. A. Dold, Fixed point index and fixed point theorem for Euclidean neighborhood retracts, Topology 4 (1965), 1-8. MR 33 \#1850.

3. E. R. Fadell, Some examples in fixed point theory, Pacific J. Math. 33 (1970), 89100. MR 41 \#6195.

4. R. P. Jerrard, Inscribed squares in plane curves, Trans. Amer. Math. Soc. 98 (1961), 234-241. MR 22 \#11354.

5. - On Knaster's conjecture, Trans. Amer. Math. Soc. 170 (1972), 385-402. MR 46 \#8212. $35 \# 2270$.

6. R. J. Knill, Cones, products and fixed points, Fund. Math. 60 (1967), 35-46. MR

7. K. Kuratowski, Problem 49, Fund. Math. 15 (1930), 356.

8. W. Lopez, An example in the fixed point theory of polyhedra, Bull. Amer. Math. Soc. 73 (1967), 922-924. MR 35 \#7323. NOIS 61801

DEPARTMENT OF MATHEMATICS, UNIVERSITY OF ILLINOIS, URBANA, ILLI- 